\title{
Nocardia amikacinitolerans and cytomegalovirus: distinctive clinical and radiological characterization of the rare etiologies of brain abscesses: report of 2 cases
}

\author{
Travis Quinoa, BS, ${ }^{1}$ Fareed Jumah, MD, ${ }^{1}$ Vinayak Narayan, MD, ${ }^{1}$ Zhenggang Xiong, MD, ${ }^{2}$ \\ Anil Nanda, MD, MPH, ${ }^{1}$ and Simon Hanft, MD${ }^{1}$
}

Departments of ${ }^{1}$ Neurosurgery and ${ }^{2}$ Pathology and Laboratory Medicine, Rutgers-Robert Wood Johnson Medical School and University Hospital, Rutgers University, New Brunswick, New Jersey

\begin{abstract}
Central nervous system infections in immunosuppressed patients are rare but potentially lethal complications that require swift diagnoses and intervention. While the differential diagnosis for new lesions on neuroradiological imaging of immunosuppressed patients typically includes infections and neoplasms, image-based heuristics to differentiate the two has been shown to have variable reliability.

The authors describe 2 rare CNS infections in immunocompromised patients with atypical physical and radiological presentations. In the first case, a 59-year-old man, who had recently undergone a renal transplantation, was found to have multifocal Nocardia amikacinitolerans abscesses masquerading as neoplasms on diffusion-weighted imaging (DWI); in the second case, a 33-year-old man with suspected recurrent Hodgkin's lymphoma was found to have a nonpyogenic abscess with cytomegalovirus (CMV) encephalitis.

As per review of the literature, this appears to be the first case of brain abscess caused by $N$. amikacinitolerans, a recently isolated superbug. Despite confirmation through brain biopsy later on in case 1 , the initial radiological appearance was atypical, showing subtle diffusion restriction on DWI. Similarly, the authors present a case of CMV encephalitis that presented as a ring-enhancing lesion, which is extremely rare. Both cases draw attention to the reliability of neuroimaging in differentiating an abscess from a neoplasm.
\end{abstract}

https://thejns.org/doi/abs/10.3171/2019.5.FOCUS19284

KEYWORDS brain abscess; Nocardia amikacinitolerans; cytomegalovirus; CMV encephalitis; CNS infection; immunocompromised

$\mathrm{C}$ ENTRAL nervous system abscesses are rare sequelae in immunosuppressed and immunocompromised states and can present a significant challenge in their management. Neuroimaging is an important tool for timely diagnosis and intervention, which are essential to improving brain abscess outcomes that can otherwise be devastating. CT and conventional MRI are inadequate for differentiating between brain abscesses and other ring-enhancing lesions, most importantly neoplasms. Therefore, functional MRI sequences such as diffusion-weighted imaging (DWI) play an important role making this distinction, where abscesses tend to restrict on DWI and exhibit low apparent diffusion coefficient (ADC) values, suggesting an underlying viscous fluid. ${ }^{7,13}$ However, this is not always the case. We present 2 cases of atypical presentations of rare brain abscesses that not only add to the literature of rare CNS infections but also highlight the importance of maintaining a high index of suspicion for infection in immunocompromised patients, despite neuroradiological findings that may suggest otherwise.

\section{Case Reports}

Case 1: Nocardia amikacinitolerans Brain Abscess History and Physical Examination

A 59-year-old male dentist presented to our emergency department with relatively sudden-onset but progressive diplopia, headache, and ataxia of 1-week duration. The double vision was relieved when covering one eye at a time. The headache was frontal, throbbing in nature, and transiently relieved by acetaminophen. The patient was evaluated by an ophthalmologist who suspected ocular palsy

ABBREVIATIONS ADC = apparent diffusion coefficient; CMV = cytomegalovirus; $\mathrm{DWI}=$ diffusion-weighted imaging; TMP/SMX = trimethoprim/sulfamethoxazole . SUBMITTED April 1, 2019. ACCEPTED May 13, 2019.

INCLUDE WHEN CITING DOI: 10.3171/2019.5.FOCUS19284. 

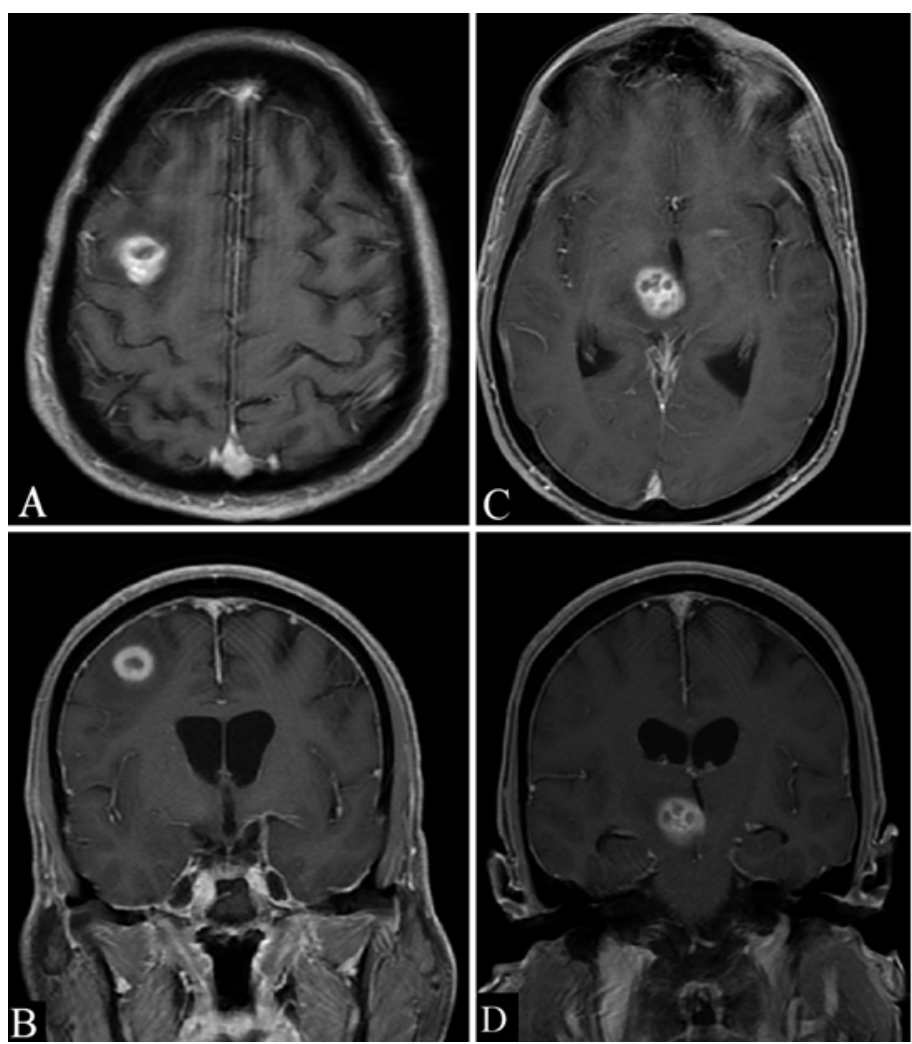
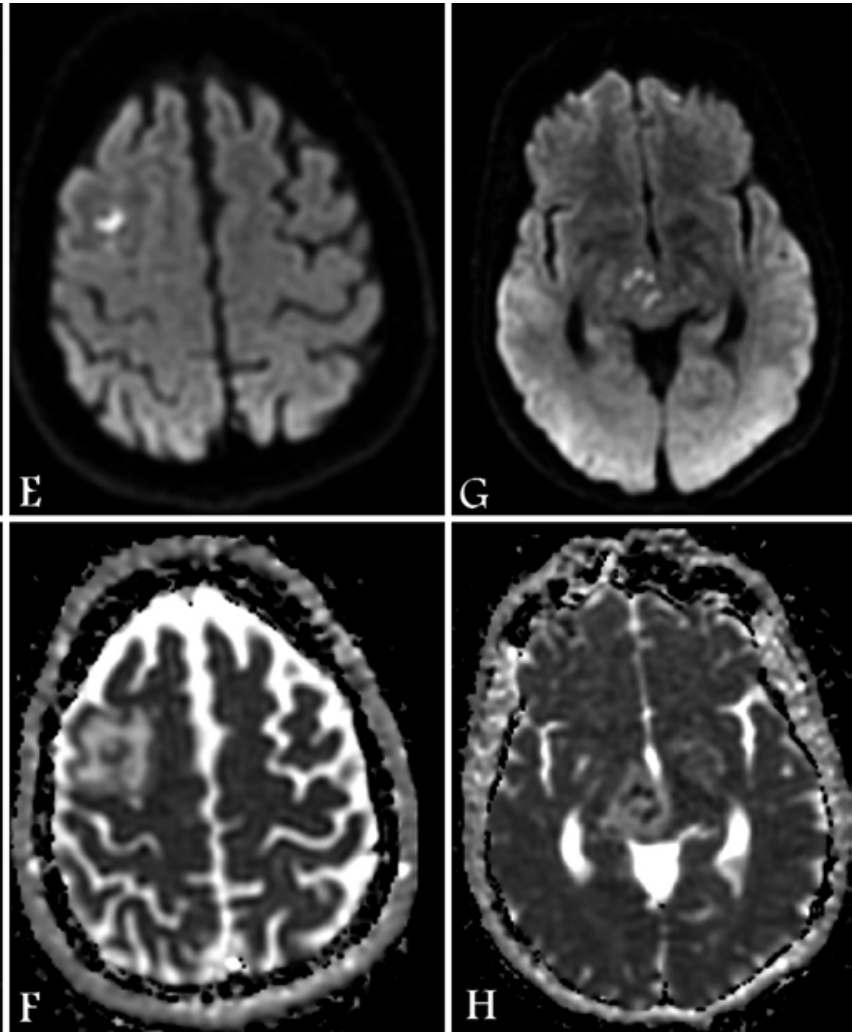

FIG. 1. Case 1. Cerebral MRI sequences taken on admission. Axial (A) and coronal (B) T1-weighted contrast-enhanced images showing a ring-enhancing mass in the right frontal lobe on sections surrounded by vasogenic edema. Axial (C) and coronal (D) T1weighted contrast-enhanced images showing the right midbrain-thalamic mass with punctate necrotic areas causing aqueductal compression and midline shift 3-4 mm to the left. Partial restricted diffusion can be seen on DWI (E) and corresponding ADC map (F) of right frontal lesion and right thalamic-midbrain lesion ( $\mathbf{G}$ and $\mathbf{H})$.

and advised the patient to see a neurologist. The patient's medical history was significant for end-stage renal disease and hemodialysis for 8 years, a renal transplant 8 weeks prior to admission, and steroid-induced diabetes mellitus and hypertension diagnosed after the transplantation. The extensive list of medications was notable for trimethoprim/ sulfamethoxazole (TMP/SMX), mycophenolate, prednisone, tacrolimus, and valganciclovir. The patient had no fatigue, fever, or weight loss and reported full adherence to medications. Physical examination showed a patient who was alert and oriented to time, place, and person, with right oculomotor nerve palsy and left facial droop. Laboratory workup was positive for normocytic normochromic anemia (hemoglobin $10.2 \mathrm{~g} / \mathrm{dl}$ ), mild thrombocytopenia (platelet count $118,000 / \mu \mathrm{l})$, and low absolute lymphocyte count $(150 / \mu l)$. The patient's white blood cell count was $8400 / \mu l$, with an absolute neutrophil count of $7200 / \mu 1$.

\section{Imaging Studies}

Noncontrast brain CT revealed a subtle right frontal patchy hypodensity with surrounding edema, in addition to a right midbrain-thalamic 15 -mm hemorrhagic lesion causing ventricular effacement and 2- to $3-\mathrm{mm}$ midline shift to the left. MRI with and without contrast showed a largely homogeneously enhancing $2-\mathrm{cm}$ mass in the right frontal subcortex and a $2-\mathrm{cm}$ right thalamic mass demonstrating heterogeneous enhancement with punctate central nonenhancing areas suggestive of necrosis (Fig. 1). Both masses were surrounded by vasogenic edema, with the thalamic mass causing a 2- to 3-mm midline shift and mild hydrocephalus due to aqueductal compression. Gradient echo MRI showed minimal hemorrhage in the thalamic mass. DWI showed subtle restricted diffusion in both lesions. The differential diagnosis was largely limited to neoplastic and infectious entities, with the most likely neoplastic lesions being either metastatic disease or lymphoma. A full-body CT scan showed no suspicious masses suggestive of widespread metastatic disease; hence, an open brain biopsy of the frontal lobe lesion was planned.

\section{Operative and Postoperative Course}

Due to the possibility of lymphoma, dexamethasone was not administered pre- or intraoperatively so as not to compromise the yield of the biopsy specimen. The lesion was approached via a standard right frontal craniotomy made just above the mass using neuronavigation. We entered the mass at its most superficial location. On gross inspection, emulsified brain tissue was seen, and a whitish viscous fluid oozed out of the friable mass, both of which were extracted for histopathology and cultures. The patient tolerated the operation without any issues. The surgical pathology report showed an inflammatory abscess 
(dense neutrophilic infiltration) with clusters of gram-positive beaded filamentous microorganisms morphologically compatible with Nocardia. Microbiological and sensitivity cultures identified Nocardia amikacinitolerans the species. The patient was started on TMP/SMX and imipenem. On follow-up 1 month after surgery, the patient reported improvement of his ataxia but not the double vision, and imaging showed persistent vasogenic edema around the old lesion in the frontal lobe-most likely postbiopsy changes-with shrinkage of midbrain-thalamic mass. His white blood cell count and absolute neutrophil count were normal (7800/ $\mu 1$ and $7300 / \mu l$, respectively) but absolute lymphocyte count $(0.31 / \mu \mathrm{l})$ and platelets $(87,000 / \mu \mathrm{l})$ remained low. The patient is currently following up with hematology and department of infectious diseases.

\section{Case 2: Nonpyogenic Abscess With Cytomegalovirus Encephalitis}

History and Examination

A 33-year-old man, with a distant history of 6 cycles of chemotherapy for Hodgkin's lymphoma and subsequent autologous bone marrow transplantation after a relapse, presented to an outside hospital with fever, cough, headaches, and lethargy that began months prior. During this time, the patient also reported a 40-pound weight loss due to decreased appetite, as well as frequent subjective fevers. At the outside hospital, workup revealed acute sinusitis and pneumonia; incidental findings included hypogammaglobulinemia, new mediastinal and bilateral hilar lymphadenopathy, mild splenomegaly, and a new right periventricular frontal lobe ring-enhancing lesion. The patient had previously been following up with an oncologist, and his lymphoma had been in remission until he was lost to follow-up in 2009. After several courses of antibiotics, the patient was transferred to our institution for neurosurgical evaluation and biopsy of the new intracranial lesion.

The patient complained of only occasional moderately severe headaches, along with improved residual symptoms of pleuritic chest pain and cough from the pneumonia. Interestingly, he had seen his optometrist within the previous 2 months for a routine evaluation, and she identified papilledema, for which she recommended brain imaging. The patient was made aware of this and did not follow-up accordingly. Physical examination revealed a slight left-sided pronator drift, left superior homonymous quadrantanopia, and blunted affect. The patient denied any gait or balance disturbance, visual complaints, sensation disturbances, or weakness. Laboratory workup was unremarkable other than a slightly elevated absolute lymphocyte count of 3500/ul (reference range 850-3000/ $\mathrm{Ll}$ ).

\section{Imaging Studies}

MRI of the brain with and without contrast revealed a deep-seated 4-cm irregular rim-enhancing lesion in the right inferior frontal lobe involving the caudate and adjacent ganglionic structures (Fig. 2). There was some mass effect displacing the optic chiasm noted in the report. Also noted was surrounding vasogenic edema that extended into the region of the corpus callosum. The mass showed a hemosiderin ring but no central restricted diffusion. The differential diagnosis suggested by the imaging included
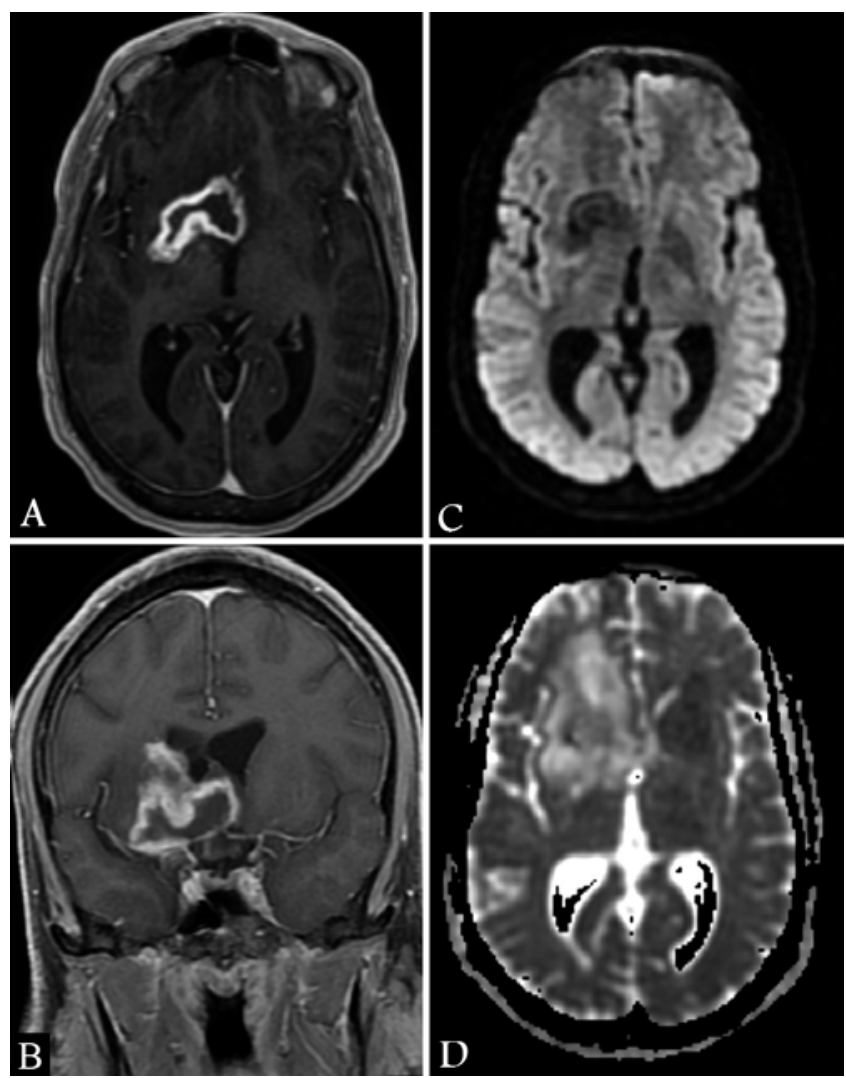

FIG. 2. Case 2. Cerebral T1-weighted contrast-enhanced MRI scans (A and $\mathbf{B}$ ) showing an irregular mass in the right ganglionic region crossing the midline and compressing the optic chiasm. No diffusion restriction is seen on DWI (C) and corresponding ADC map (D).

a neoplasm, hematoma, or atypical nonpyogenic abscess with encephalitis. Repeat MRI with Stealth protocol was performed at this institution and confirmed the findings.

\section{Operative and Postoperative Course}

After the patient was counseled regarding the initial findings on brain imaging, he agreed to undergo a stereotactic needle biopsy of the mass. The needle biopsy was recommended because of the high-risk location of the mass and the diagnostic uncertainty. In the operating room, a right frontal trajectory was chosen to access the mass. Then using MRI neuronavigation, a biopsy needle was passed along the planned trajectory path into the lesion. Examination of a specimen taken from this approach revealed grossly abnormal tissue. Several samples were taken, and evaluation of frozen sections confirmed obvious lesional tissue. The patient tolerated the procedure well and was medically cleared for discharge the following day. The pathology report on the specimens collected during the biopsy revealed patchy dense lymphoid infiltrate composed of small, mature cells without overt signs of atypia. No necrosis was identified. The overall features of the sample were consistent with a chronic inflammatory infiltrate without evidence of any lymphoproliferative disorder. Neuropathology revealed scattered enlarged neuroglial cells with viral inclusions that stained immunologically positive for cytomegalovirus (CMV) (Figs. 3 and 4). 


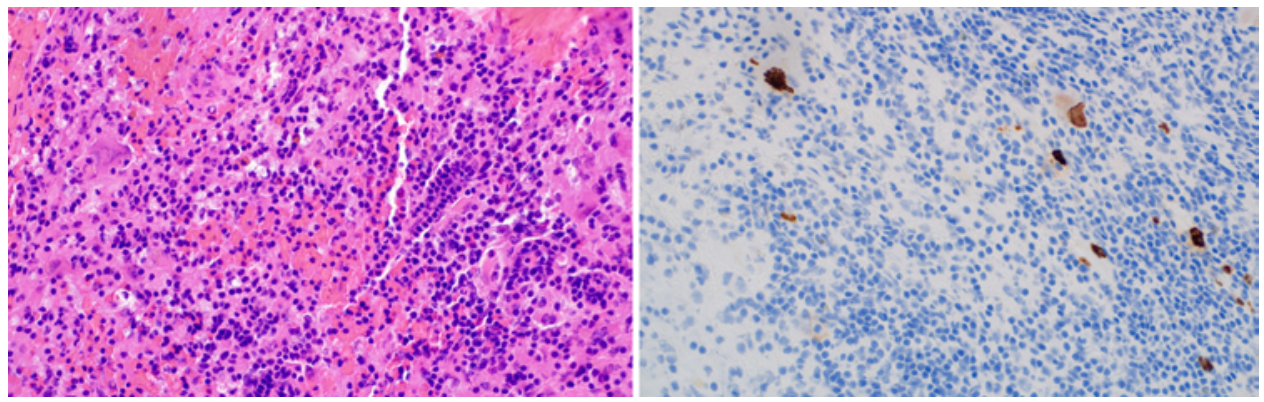

FIG. 3. Case 2. Left: Photomicrograph showing extensive inflammation with mixed population of lymphocytes, plasma cells, and neutrophils. Scattered enlarged neuroglial cells with intranuclear inclusions were present. H \& E, original magnification $\times 100$. Right: Immunostaining with anti-CMV antibody was positive and confirmed a CMV infection. Original magnification ×100.

During an outpatient follow-up visit 3 weeks after surgery, the patient was clinically stable and neurologically intact. He was referred to infectious diseases for management of the CMV infection, where he was started on a regimen of oral valganciclovir and scheduled for follow-up brain MRI.

\section{Discussion}

Despite advances in imaging, neurosurgical technique, and antimicrobial therapy, brain abscesses remain a challenging entity that are often associated with a grim prognosis. They typically affect individuals with predisposing factors like immunocompromise (e.g., HIV, hematopoietic malignancy, chronic steroid use, and, more recently, a growing litany of immunosuppressive medications), breach of the blood-brain barrier (e.g., due to cranial surgery or sinusitis), or a systemic source of infection (e.g., septic emboli of infective endocarditis). Pathogens can access the brain through contiguous spread in roughly $50 \%$ of cases, hematogenous seeding in about $33 \%$, and an unknown mechanism in the remaining cases. ${ }^{8}$ The pathological process begins with a focus of cerebritis that evolves into a purulent lesion surrounded by a well-vascularized fibrotic capsule. ${ }^{26}$ Patients with brain abscesses can present with any number of symptoms. Headache, fever, and focal neurological deficits are among the most common presenting symptoms, but they are infrequently seen together.'

There have been a number of abscess-causing organisms described in the literature. In 2014, Brouwer and colleagues published a systematic review and meta-analysis of the clinical characteristics, causal organisms, and outcomes of brain abscesses in 123 studies from 1935 to $2012 .{ }^{8}$ Their analysis of 9699 cases revealed a total of 85 different causal organisms. Most commonly among them were Streptococcus and Staphylococcus spp., which comprised $34 \%$ and $18 \%$ of the cases analyzed, respectively. Of the less frequently reported causal organisms in the meta-analysis, Nocardia, Enterococcus, Mycobacterium tuberculosis, parasites, and fungi were the most rare. As the authors pointed out, $86 \%$ of the cases in their analysis shared the characteristic of an underlying predisposing condition like immunocompromise or distant primary infectious foci. ${ }^{8}$ As might be expected, patients with the predisposing condition of an underlying immunocompromised state are at an increased risk of developing a brain abscess with a rare causal organism..$^{8,9}$

Some insight as to the causal organism can be gleaned from neuroradiological presentation. For example, parasitic abscess typically presents as small multifocal lesions, whereas bacterial and fungal abscesses tend to be larger, singular, or few in number. ${ }^{9}$ Recent investigations into susceptibility-weighted MRI sequences have been helpful in identifying differentiating characteristics between bacterial and fungal abscesses. ${ }^{3}$

As will be discussed, neuroradiological techniques can be useful for differentiating an abscess from other etiologies of intracranial mass lesions. In our case series, we presented 2 unique cases. The first case involved a pyogenic abscess exhibiting unusual radiological features on DWI,

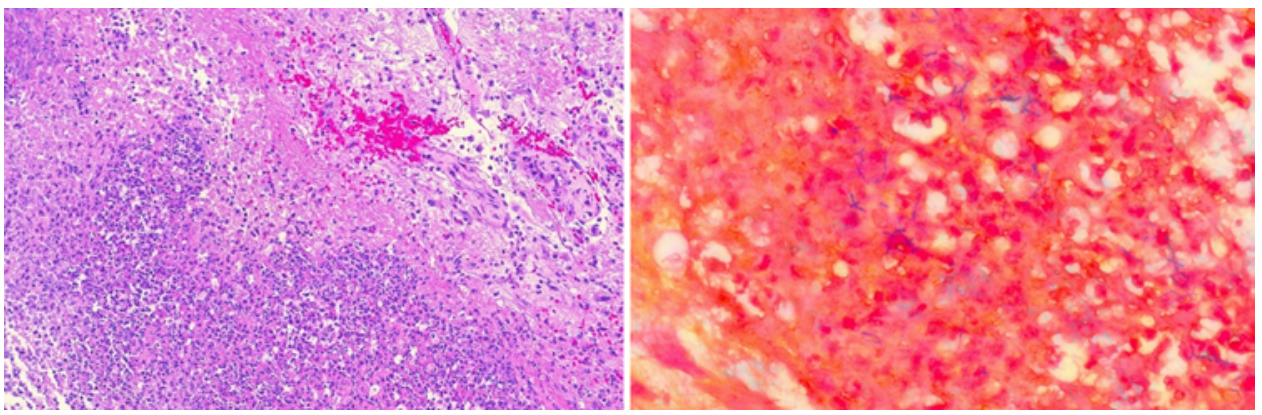

FIG. 4. Case 2. Left: Photomicrograph showing extensive inflammation with predominant neutrophils. H \& E, original magnification $\times 40$. Right: Gram staining identified positively stained rod-shaped bacteria. Original magnification $\times 400$. 
and the second involved CMV encephalitis presenting as a focal abscess, another extremely rare presentation. Our aim here is to increase awareness of these rare intracranial pathogens and discuss the reliability of state-of-the-art neuroimaging in differentiating between intracranial abscesses and neoplasms. Together, these cases demonstrate the need to prioritize an abscess in the differential diagnosis of new intracranial mass lesions in patients with predisposing factors such as an immunocompromised state and also, when an abscess is suspected in these patients, to consider both common and unlikely casual agents.

\section{Nocardia amikacinitolerans}

Nocardia is a rare bacterial opportunistic pathogen, responsible for around $1 \%-2 \%$ of all brain abscesses, ${ }^{29}$ with a reported mortality rate reaching up to $66 \%$ compared with $<10 \%$ in other bacterial abscesses. ${ }^{33}$ The abscesses are weakly acid-fast, gram-positive, branching filamentous aerobes found ubiquitously in the soil all over the planet. The most common infectious culprits in humans are $N$. asteroides, $N$. brasiliensis, and $N$. caviae. Nocardia can enter the body through inhalation or direct skin inoculation, which usually causes a self-limiting asymptomatic infection that goes unnoticed in the immunocompetent individual. However, immunocompromised states (e.g., post-organ transplant as in our patient) allow primary infection to propagate into systemic bacteremia, which has a particular affinity to spread to neural tissue. CNS involvement has been observed in almost half of disseminated nocardiosis cases. ${ }^{2,5}$

In 2013, Ezeoke et al. ${ }^{17}$ isolated a novel species of Nocardia from eye and lung sources in 5 patients. Antimicrobial susceptibility testing revealed that all 5 isolates were resistant to amikacin, ciprofloxacin, and clarithromycin, with amikacin resistance being the highest. Interestingly, resistance to amikacin among people with Nocardia is rare, ${ }^{10,12,49}$ making it a differentiating characteristic for this species, hence the nomenclature. We did not find any previous reports of infection with this species. The choice of appropriate antibiotic for this species can be considered between amoxicillin/clavulanate, ceftriaxone, imipenem, linezolid, and TMP/SMX. In our patient, a combination of TMP/SMX and imipenem seemed efficacious given the radiologically proven shrinkage of the thalamic lesions on sequential MR images.

\section{The Role of Neuroimaging in Brain Abscesses}

Brain abscesses appear as ring-enhancing lesions, with a hypointense rim on T2-weighted MRI being a common feature shared with necrotic glioblastomas. ${ }^{19}$ However, conventional MRI alone can be inadequate in differentiating an abscess from other intracranial pathologies, especially neoplasms. This is further compounded by the frequent absence of reliable symptoms in brain abscess like fever and altered mental status. ${ }^{8}$ Therefore, MRI sequences such as DWI and ADC maps are considered most telling in the case of cerebral abscess. Brain abscesses usually restrict on DWI with corresponding low ADC values; this leads to the characteristic "white" appearance on DWI images and "dark" appearance on ADC maps. In our case, however, the abscess showed only subtle diffusion restriction on DWI, which gave the initial impression of the second most likely diagnosis, brain metastases. Open biopsy later confirmed a brain abscess. This raises the important question as to whether certain abscesses fail to cause characteristic DWI restriction and whether this failure is based on organism type or perhaps the age or state of the abscess. In light of our case, we reviewed the literature for reported nocardial abscess appearance on DWI. Table 1 demonstrates that $10(59 \%)$ of 17 cases

TABLE 1. Appearance of nocardial brain abscesses on DWI and ADC maps reported in the literature

\begin{tabular}{|c|c|c|c|c|c|}
\hline Authors \& Year & $\begin{array}{l}\text { No. of } \\
\text { Cases }\end{array}$ & $\begin{array}{l}\text { Age (yrs), } \\
\text { Sex }\end{array}$ & Nocardial Species & Capsule on DWI & ADC Maps \\
\hline Yamada et al., 2005 & 1 & $58, F$ & NS & Heterogeneous & NS \\
\hline Zakaria et al., 2008 & 1 & $40, \mathrm{M}$ & N. asteroides & Hyperintense & NS \\
\hline Hashimoto et al., 2008 & & $77, \mathrm{~F}$ & N. nova & Hyperintense & Low \\
\hline Roca \& Merino, 2010 & 1 & $33, \mathrm{M}$ & N. nova & Hyperintense & NS \\
\hline Pyatigorskaya et al., 2010 & 1 & $59, \mathrm{NS}$ & N. abscessus & Hyperintense & Low \\
\hline Alijani et al., 2013 & 1 & $31, F$ & NS & Hyperintense & NS \\
\hline Kranick \& Zerbe, 2013 & 1 & $18, \mathrm{M}$ & N. transvalensis & Hyperintense & Low \\
\hline Nandhagopal et al., 2014 & 1 & $16, \mathrm{M}$ & NS & Hyperintense & Low \\
\hline Pamukçuoğlu et al., 2014 & 2 & $61, \mathrm{~F} ; 60, \mathrm{~F}$ & N. cyriacigeorgica & Hyperintense; hyperintense & Low; low \\
\hline Beuret et al., 2015 & 2 & $64, \mathrm{~F} ; 68, \mathrm{M}$ & N. farcinica & Hyperintense; hyperintense & High; low \\
\hline Molière \& Krémer, 2015 & 1 & $65, \mathrm{~F}$ & N. novia & Hyperintense & Low \\
\hline Stefaniak, 2015 & 1 & $59, \mathrm{M}$ & NS & Hyperintense & NS \\
\hline Majeed et al., 2017 & 1 & $72, \mathrm{M}$ & N. kroppenstedtii & Hyperintense & Low \\
\hline Chaudhari et al., 2017 & 1 & $75, \mathrm{M}$ & N. farcinica & Hyperintense & Low \\
\hline Yamamoto et al., 2017 & 1 & $73, \mathrm{M}$ & N. araoensis & Hyperintense & Isointense \\
\hline Present case & 1 & $59, \mathrm{M}$ & N. amikacinitolerans & Heterogeneous & Heterogeneous \\
\hline
\end{tabular}




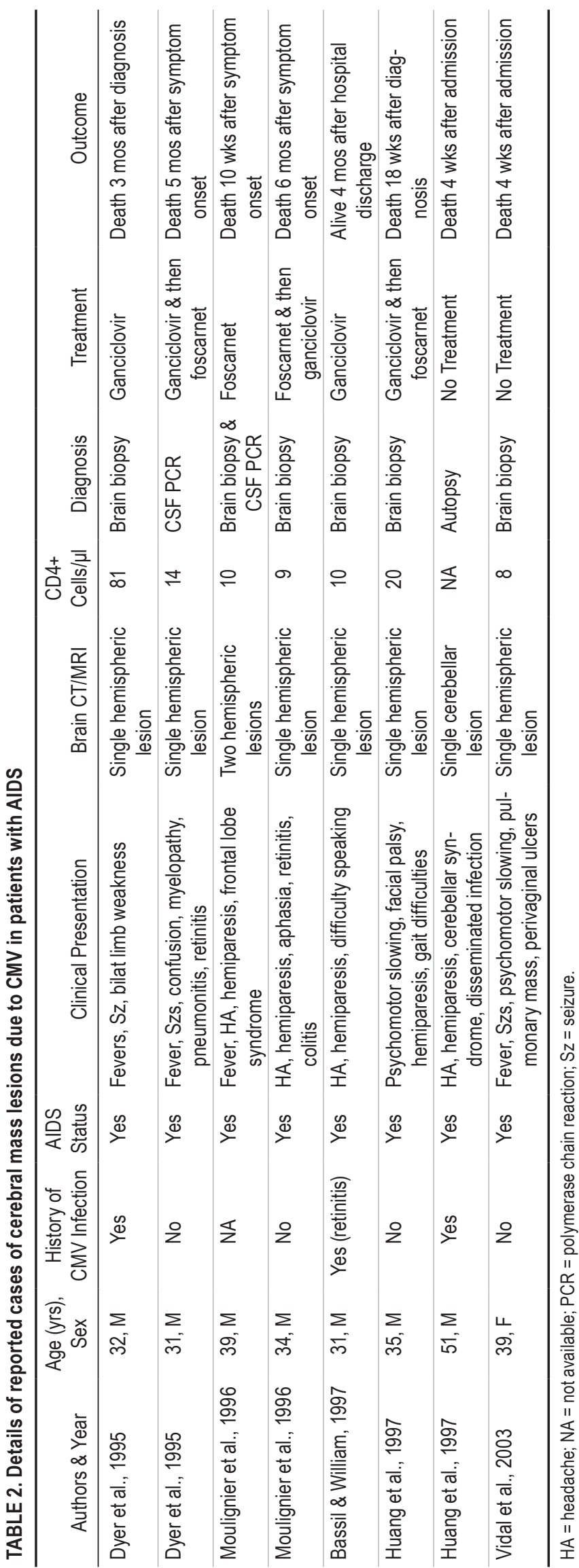

of nocardial brain abscesses showed true restricted diffusion, i.e., hyperintensity on DWI and low signal on ADC maps. $1,6,11,21,27,32,36,39,41,42,46,47,51-54$

High signal intensity on DWI with low ADC values for cerebral abscesses was first reported by Ebisu et al., ${ }^{15}$ with many subsequent studies reporting similar findings. ${ }^{18,20}$ In a meta-analysis Xu et al. ${ }^{50}$ found that DWI had a high sensitivity (0.95) and specificity (0.94) for differentiating brain abscesses from other cystic brain masses, showing it is a reliable method in detecting pyogenic brain abscesses. However, there are exceptions. Cases that have involved absent diffusion restriction on DWI have been reported before in patients with brain abscesses. ${ }^{28,40}$ This unusual radiological appearance could be attributed to several factors that can alter pus composition and viscosity, such as early initiation of antimicrobial therapy, failure of adequate neutrophilic infiltration due to immunocompromise, the age of abscess, and even the type of infecting organism. ${ }^{35}$ For example, hypointensity and heterogeneous appearance on DWI have been reported with fungal and tuberculous abscesses. ${ }^{31,38}$

\section{Encephalitis}

CMV is a Herpesviridae virus that is common in the population as a latent infection. Primary infection with CMV is typically subclinical and becomes latent in the immunocompetent individual. Reactivation of dormant CMV is more frequent in critically ill patients and can manifest as a systemic or end-organ disease like pneumonitis or gastroenteritis. ${ }^{30,43}$ While in the past CMV recurrence or infection was most typically seen in patients with AIDS, tissue-invasive CMV infection has had an increasing incidence among individuals who have undergone solid-organ transplant, hematopoietic stem cell transplant, and other immunosuppressed or immunocompromised states. ${ }^{16,22,30,43}$

CMV brain infection, most commonly manifesting as encephalitis or ventriculitis, is a rare and deadly complication in immunocompromised patients and requires immediate treatment. Because of the high mortality and morbidity rates, current recommendations suggest proactive screening for CMV and treatment for anyone with a detectable serological or CSF viral load. ${ }^{16,22,34,43}$ Typical findings on MRI that suggest CNS disease include white matter nodular signal abnormalities, ventriculitis with accompanying subependymal lesions on T2-weighted FLAIR images, the classic "Owl's sign" seen in CMV ventriculitis, and, rarely, a focal mass lesion. ${ }^{45}$ There is a paucity of data regarding DWI findings, and, as suggested elsewhere, this is likely because DWI was not routinely available in the era before highly active antiretroviral therapy ${ }^{45}$ Overall, CMV recurrence typically carries a poor prognosis, and therapeutic options are limited to only a few antiviral agents.

Interestingly, the CMV brain abscess in our patient was incidentally discovered during workup for acute sinusitis, which revealed the right ganglionic lesion. The incidence of CMV encephalitis, especially in a patient without AIDS, is extremely rare. Moreover, there are only 8 previously documented cases of intracranial CMV masses, all of which occurred in patients with significantly low CD4-positive T-lymphocyte counts (8-81 cells/ $\mu \mathrm{l}), 4,14,23,37,48$ As shown in Table $2,{ }^{48}$ all these patients had AIDS, were significantly symptomatic, and died soon after diagnosis. Characteristics of lesions on MRI were also similar to those of our 
patient's: focal, most often single, supratentorial ring-enhancing lesions that could not be distinguished as either neoplasm or infection. Unfortunately, T- and B-cell counts were never measured during the patient's hospitalization; however, the patient has multiple risk factors for CMV recurrence: possible cellular immunosuppression secondary to recurrent lymphoma, known hypogammaglobulinemia, and a history of autologous hematopoietic stem cell transplantation. The pathophysiology behind mass lesion development is not well understood, but immunoglobulin deficiency, especially in bone marrow transplant recipients, may play a role. ${ }^{24,25,44}$

\section{Conclusions}

Nocardia amikacinitolerans is a newly isolated opportunistic bacterial superbug that is highly resistant to amikacin but is sensitive to TMP-SMX and imipenem, among others. Functional MRI sequences play a fundamental role in the timely diagnosis and management of brain abscesses. True restricted diffusion-defined as hyperintensity on DWI and low signal on ADC maps - is an important clue to distinguish brain abscesses from other ring-enhancing lesions. However, the absence of diffusion restriction on DWI does not preclude the possibility of an abscess, which should stay high on the list of differential diagnoses, especially in an immunocompromised host. Furthermore, we describe the ninth reported case of intracranial CMV abscess in a patient with suspected recurrent lymphoma-the first case in a patient without AIDS. These 2 case studies emphasize the importance and limitations of neuroimaging in the diagnosis of CNS infections and the need to consider rare causal agents in immunocompromised patients.

\section{References}

1. Alijani N, Mahmoudzadeh S, Hedayat Yaghoobi M, Geramishoar M, Jafari S: Multiple brain abscesses due to nocardia in an immunocompetent patient. Arch Iran Med 16:192194,2013

2. Anagnostou T, Arvanitis M, Kourkoumpetis TK, Desalermos A, Carneiro HA, Mylonakis E: Nocardiosis of the central nervous system: experience from a general hospital and review of 84 cases from the literature. Medicine (Baltimore) 93:19-32, 2014

3. Antulov R, Dolic K, Fruehwald-Pallamar J, Miletic D, Thurnher MM: Differentiation of pyogenic and fungal brain abscesses with susceptibility-weighted MR sequences. Neuroradiology 56:937-945, 2014

4. Bassil HF, William DC: Cytomegalovirus encephalitis in an HIV positive patient presenting with a cerebral mass lesion. AIDS Patient Care STDS 11:319-321, 1997

5. Beaman BL, Beaman L: Nocardia species: host-parasite relationships. Clin Microbiol Rev 7:213-264, 1994

6. Beuret F, Schmitt E, Planel S, Lesanne G, Bracard S: Subtentorial cerebral nocardiosis in immunocompetent patients: CT and MR imaging findings. Diagn Interv Imaging 96:953957, 2015

7. Britt RH, Enzmann DR: Clinical stages of human brain abscesses on serial CT scans after contrast infusion. Computerized tomographic, neuropathological, and clinical correlations. J Neurosurg 59:972-989, 1983

8. Brouwer MC, Coutinho JM, van de Beek D: Clinical characteristics and outcome of brain abscess: systematic review and meta-analysis. Neurology 82:806-813, 2014
9. Brouwer MC, van de Beek D: Epidemiology, diagnosis, and treatment of brain abscesses. Curr Opin Infect Dis 30:129134,2017

10. Brown-Elliott BA, Brown JM, Conville PS, Wallace RJ Jr: Clinical and laboratory features of the Nocardia spp. based on current molecular taxonomy. Clin Microbiol Rev 19:259282,2006

11. Chaudhari DM, Renjen PN, Sardana R, Butta H: Nocardia farcinica brain abscess in an immunocompetent old patient: a case report and review of literature. Ann Indian Acad Neurol 20:399-402, 2017

12. Conville PS, Brown JM, Steigerwalt AG, Brown-Elliott BA, Witebsky FG: Nocardia wallacei sp. nov. and Nocardia blacklockiae sp. nov., human pathogens and members of the "Nocardia transvalensis Complex." J Clin Microbiol 46:1178-1184, 2008

13. Desprechins B, Stadnik T, Koerts G, Shabana W, Breucq C, Osteaux M: Use of diffusion-weighted MR imaging in differential diagnosis between intracerebral necrotic tumors and cerebral abscesses. AJNR Am J Neuroradiol 20:1252-1257, 1999

14. Dyer JR, French MA, Mallal SA: Cerebral mass lesions due to cytomegalovirus in patients with AIDS: report of two cases. J Infect 30:147-151, 1995

15. Ebisu T, Tanaka C, Umeda M, Kitamura M, Naruse S, Higuchi T, et al: Discrimination of brain abscess from necrotic or cystic tumors by diffusion-weighted echo planar imaging. Magn Reson Imaging 14:1113-1116, 1996

16. Emery V, Zuckerman M, Jackson G, Aitken C, Osman H, Pagliuca A, et al: Management of cytomegalovirus infection in haemopoietic stem cell transplantation. Br J Haematol 162:25-39, 2013

17. Ezeoke I, Klenk HP, Pötter G, Schumann P, Moser BD, Lasker BA, et al: Nocardia amikacinitolerans sp. nov., an amikacin-resistant human pathogen. Int J Syst Evol Microbiol 63:1056-1061, 2013

18. Guzman R, Barth A, Lövblad KO, El-Koussy M, Weis J, Schroth G, et al: Use of diffusion-weighted magnetic resonance imaging in differentiating purulent brain processes from cystic brain tumors. J Neurosurg 97:1101-1107, 2002

19. Haimes AB, Zimmerman RD, Morgello S, Weingarten K, Becker RD, Jennis R, et al: MR imaging of brain abscesses. AJR Am J Roentgenol 152:1073-1085, 1989

20. Hartmann M, Jansen O, Heiland S, Sommer C, Münkel K, Sartor K: Restricted diffusion within ring enhancement is not pathognomonic for brain abscess. AJNR Am J Neuroradiol 22:1738-1742, 2001

21. Hashimoto M, Johkura K, Ichikawa T, Shinonaga M: Brain abscess caused by Nocardia nova. J Clin Neurosci 15:87-89, 2008

22. Hebart H, Schröder A, Löffler J, Klingebiel T, Martin H, Wassmann B, et al: Cytomegalovirus monitoring by polymerase chain reaction of whole blood samples from patients undergoing autologous bone marrow or peripheral blood progenitor cell transplantation. J Infect Dis 175:1490-1493, 1997

23. Huang PP, McMeeking AA, Stempien MJ, Zagzag D: Cytomegalovirus disease presenting as a focal brain mass: report of two cases. Neurosurgery 40:1074-1079, 1997

24. Hyde R, Chertoff JL, Ataya A: Diffuse pulmonary nodulesan unusual case of CMV pneumonitis. Am J Respir Crit Care Med 197:A5445, 2018

25. Kauffman CA, Linnemann CC Jr, Alvira MM: Cytomegalovirus encephalitis associated with thymoma and immunoglobulin deficiency. Am J Med 67:724-728, 1979

26. Kielian T: Immunopathogenesis of brain abscess. J Neuroinflammation 1:16, 2004

27. Kranick SM, Zerbe CS: Case report from the NIH Clinical Center: CNS nocardiosis. J Neurovirol 19:505-507, 2013 
28. Lee EJ, Ahn KJ, Ha YS, Oh HE, Park CS, Song SY, et al: Unusual findings in cerebral abscess: report of two cases. Br J Radiol 79:e156-e161, 2006

29. Lin YJ, Yang KY, Ho JT, Lee TC, Wang HC, Su FW: Nocardial brain abscess. J Clin Neurosci 17:250-253, 2010

30. Ljungman P, Boeckh M, Hirsch HH, Josephson F, Lundgren J, Nichols G, et al: Definitions of cytomegalovirus infection and disease in transplant patients for use in clinical trials. Clin Infect Dis 64:87-91, 2017

31. Luthra G, Parihar A, Nath K, Jaiswal S, Prasad KN, Husain $\mathrm{N}$, et al: Comparative evaluation of fungal, tubercular, and pyogenic brain abscesses with conventional and diffusion MR imaging and proton MR spectroscopy. AJNR Am J Neuroradiol 28:1332-1338, 2007

32. Majeed A, Abdullah HM, Ullah W, Al Mohajer M: First reported case of disseminated Nocardia kroppenstedtii sp nov. infection presenting with brain abscess and endocarditis in an immunocompromised patient with mantle cell lymphoma: challenges in diagnosis and treatment. BMJ Case Rep 2017:bcr2016217337, 2017

33. Mamelak AN, Obana WG, Flaherty JF, Rosenblum ML: Nocardial brain abscess: treatment strategies and factors influencing outcome. Neurosurgery 35:622-631, 1994

34. Mengarelli A, Annibali O, Pimpinelli F, Riva E, Gumenyuk $\mathrm{S}$, Renzi D, et al: Prospective surveillance vs clinically driven approach for CMV reactivation after autologous stem cell transplant. J Infect 72:265-268, 2016

35. Mishra AM, Gupta RK, Jaggi RS, Reddy JS, Jha DK, Husain $\mathrm{N}$, et al: Role of diffusion-weighted imaging and in vivo proton magnetic resonance spectroscopy in the differential diagnosis of ring-enhancing intracranial cystic mass lesions. J Comput Assist Tomogr 28:540-547, 2004

36. Molière $\mathrm{S}$, Krémer $\mathrm{S}$ : When meningioma becomes an emergency: nocardial brain abscesses superimposed on meningioma. J Neuroradiol 42:249-251, 2015

37. Moulignier A, Mikol J, Gonzalez-Canali G, Polivka M, Pialoux G, Welker Y, et al: AIDS-associated cytomegalovirus infection mimicking central nervous system tumors: a diagnostic challenge. Clin Infect Dis 22:626-631, 1996

38. Mueller-Mang C, Castillo M, Mang TG, Cartes-Zumelzu F, Weber M, Thurnher MM: Fungal versus bacterial brain abscesses: is diffusion-weighted MR imaging a useful tool in the differential diagnosis? Neuroradiology 49:651-657, 2007

39. Nandhagopal R, Al-Muharrmi Z, Balkhair A: Nocardia brain abscess. QJM 107:1041-1042, 2014

40. Ozbayrak M, Ulus OS, Berkman MZ, Kocagoz S, Karaarslan E: Atypical pyogenic brain abscess evaluation by diffusionweighted imaging: diagnosis with multimodality MR imaging. Jpn J Radiol 33:668-671, 2015

41. Pamukçuoğlu M, Emmez H, Tunçcan OG, Oner AY, Cirak MY, Senol E, et al: Brain abscess caused by Nocardia cyriacigeorgica in two patients with multiple myeloma: novel agents, new spectrum of infections. Hematology 19:158-162, 2014

42. Pyatigorskaya N, Brugieres P, Hodel J, Mekontso Dessap A, Gaston A: What is your diagnosis? Nocardia abscessus infection. J Neuroradiol 37:192-195, 2010

43. Razonable RR, Humar A: Cytomegalovirus in solid organ transplantation. Am J Transplant 13 (Suppl 4):93-106, 2013
44. Reed EC, Bowden RA, Dandliker PS, Lilleby KE, Meyers JD: Treatment of cytomegalovirus pneumonia with ganciclovir and intravenous cytomegalovirus immunoglobulin in patients with bone marrow transplants. Ann Intern Med 109:783-788, 1988

45. Renard T, Daumas-Duport B, Auffray-Calvier E, Bourcier $\mathrm{R}$, Desal H: Cytomegalovirus encephalitis: undescribed diffusion-weighted imaging characteristics. Original aspects of cases extracted from a retrospective study, and from literature review. J Neuroradiol 43:371-377, 2016

46. Roca B, Merino J: Nocardia brain abscess. Int J Infect Dis 14 (Suppl 3):e383-e384, 2010

47. Stefaniak J: HIV/AIDS presenting with stroke-like features caused by cerebral Nocardia abscesses: a case report. BMC Neurol 15:183, 2015

48. Vidal JE, Dauar RF, Penalva de Oliveira AC, Coelho JF, Lins DL: Cerebral mass lesion due to cytomegalovirus in a patient with AIDS: case report and literature review. Rev Inst Med Trop São Paulo 45:333-337, 2003

49. Wilson RW, Steingrube VA, Brown BA, Blacklock Z, Jost $\mathrm{KC} \mathrm{Jr}, \mathrm{McNabb} \mathrm{A}$, et al: Recognition of a Nocardia transvalensis complex by resistance to aminoglycosides, including amikacin, and PCR-restriction fragment length polymorphism analysis. J Clin Microbiol 35:2235-2242, 1997

50. Xu XX, Li B, Yang HF, Du Y, Li Y, Wang WX, et al: Can diffusion-weighted imaging be used to differentiate brain abscess from other ring-enhancing brain lesions? A metaanalysis. Clin Radiol 69:909-915, 2014

51. Yamada SM, Nakai E, Toyonaga S, Nakabayashi H, Park $\mathrm{KC}$, Shimizu K: A rapidly enlarging nocardial brain abscess mimicking malignant glioma. J Nippon Med Sch 72:308311, 2005

52. Yamamoto F, Yamashita S, Kawano H, Tanigawa T, Mihara Y, Gonoi T, et al: Meningitis and ventriculitis due to Nocardia araoensis infection. Intern Med 56:853-859, 2017

53. Yeung HH: What's your diagnosis? J Pediatr Ophthalmol Strabismus 47:202, 219, 2010

54. Zakaria A, Elwatidy S, Elgamal E: Nocardia brain abscess: severe CNS infection that needs aggressive management; case report. Acta Neurochir (Wien) 150:1097-1101, 2008

\section{Disclosures}

The authors report no conflict of interest concerning the materials or methods used in this study or the findings specified in this paper.

\section{Author Contributions}

Conception and design: Hanft, Quinoa, Narayan. Acquisition of data: Quinoa, Jumah, Xiong, Nanda. Drafting the article: all authors. Critically revising the article: Hanft, Jumah, Narayan, Xiong, Nanda. Reviewed submitted version of manuscript: all authors. Approved the final version of the manuscript on behalf of all authors: Hanft. Administrative/technical/material support: Nanda. Study supervision: Hanft.

\section{Correspondence}

Simon Hanft: Rutgers-Robert Wood Johnson Medical School University Hospital, New Brunswick, NJ. sihanft@gmail.com. 